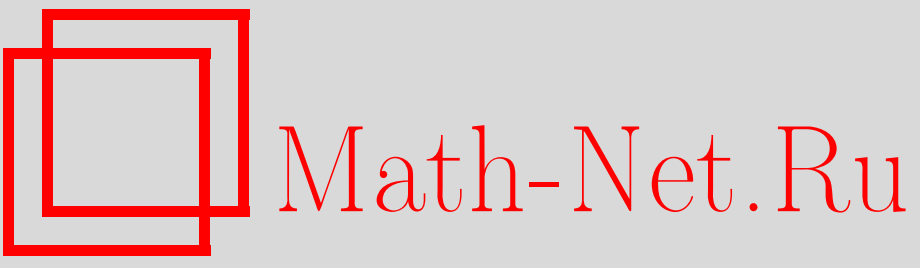

Sh. A. Ayupov, A. A. Zhalilov, Asymptotic distribution of hitting times for critical maps of the circle, Vestn. Udmurtsk. Univ. Mat. Mekh. Komp. Nauki, 2021, Volume 31, Issue 3, 365-383

DOI: https://doi.org/10.35634/vm210302

Use of the all-Russian mathematical portal Math-Net.Ru implies that you have read and agreed to these terms of use

http: //www.mathnet.ru/eng/agreement

Download details:

IP : 3.85 .183 .62

April 26, 2023, 14:29:48 
MSC2020: 37A05, 28D05

(C) Sh. A. Ayupov, A. A. Zhalilov

\section{ASYMPTOTIC DISTRIBUTION OF HITTING TIMES FOR CRITICAL MAPS OF THE CIRCLE}

It is well known that the renormalization group transformation $\mathcal{R}$ has a unique fixed point $f_{c r}$ in the space of critical $C^{3}$-circle homeomorphisms with one cubic critical point $x_{c r}$ and the golden mean rotation number $\bar{\rho}:=\frac{\sqrt{5}-1}{2}$. Denote by $\operatorname{Cr}(\bar{\rho})$ the set of all critical circle maps $C^{1}$-conjugated to $f_{c r}$. Let $f \in \operatorname{Cr}(\bar{\rho})$ and let $\mu:=\mu_{f}$ be the unique probability invariant measure of $f$. Fix $\theta \in(0,1)$. For each $n \geq 1$ define $c_{n}:=c_{n}(\theta)$ such that $\mu\left(\left[x_{c r}, c_{n}\right]\right)=\theta \cdot \mu\left(\left[x_{c r}, f^{q_{n}}\left(x_{c r}\right)\right]\right)$, where $q_{n}$ is the first return time of the linear rotation $f_{\bar{\rho}}$. We study convergence in law of rescaled point process of time hitting. We show that the limit distribution is singular w. r.t. the Lebesgue measure.

Keywords: circle homeomorphism, critical point, rotation number, hitting time, thermodynamic formalism.

DOI: $10.35634 / \mathrm{vm} 210302$

\section{Introduction}

The goal of this paper is to study the asymptotic behaviour of critical circle maps with a single critical point having an odd type. These maps have been a subject of intensive study since the early 1980's as one of the two main examples of universality in transition to chaos [1-4].

An important one-parameter family of examples of critical circle maps are the Arnold's maps defined by

$$
\mathbf{A}_{t}(x):=x+t-\frac{1}{2 \pi} \sin 2 \pi x \bmod 1, \quad x \in S^{1} .
$$

For every $t \in[0,1)$ the map $f_{t}$ is a critical map with critical point 0 of cubic type.

Following to the work of Ostlund and etc [2] we define a set of real-analytic commuting pairs that corresponds to a set of real-analytic critical circle homeomorphisms of the third order. Consider the set $\mathcal{X}_{c r}$ of pairs $(\xi, \eta)$ of real-analytic, strictly increasing on real line and satisfying the following conditions [2]:

$\left(c_{1}\right) 0<\xi(0)<1, \xi(0)=\eta(0)+1 ;$

$\left(c_{2}\right) \xi(\eta(0))=\eta(\xi(0))>0 ;$

$\left(c_{3}\right) \xi^{\prime}(0)=\eta^{\prime}(0)=\xi^{\prime \prime}(0)=\eta^{\prime \prime}(0)=0$, but $\xi^{\prime \prime \prime}(0)$ and $\eta^{\prime \prime \prime}(0)$ are non zero;

$\left(c_{4}\right)(\xi \circ \eta)^{\prime \prime \prime}(0)=(\eta \circ \xi)^{\prime \prime \prime}(0)$.

Conditions $\left(c_{1}\right)$ and $\left(c_{2}\right)$ permit us to associate a homeomorphism $f=f_{\xi, \eta}$ on the unit circle with each $(\xi, \eta) \in \mathcal{X}_{c r}$. Define $f=\xi$ on $[\eta(0), 0]$ and $f=\eta$ on $[0, \xi(0)]$ and associate the unit interval $[\eta(0), \xi(0)]$ with the circle by identifying end points. A rotation number $\rho=\rho\left(f_{\xi, \eta}\right)$ can be defined for $f_{\xi, \eta}$ in the usual way (see, for instance, [5]).

We denote by $\mathcal{X}_{c r}(\bar{\rho})$ the subset $\mathcal{X}_{c r}$ of pairs $(\xi, \eta)$ for which the rotation number $\rho\left(f_{\xi, \eta}\right)=$ $=\bar{\rho}=\frac{\sqrt{5}-1}{2}$, i. e. it is equal to the golden mean. 
Next we define the renormalization group transformation $\mathcal{R}: \mathcal{X}_{c r}(\bar{\rho}) \rightarrow \mathcal{X}_{c r}(\bar{\rho})$ (see [2]):

$$
\mathcal{R}(\xi, \eta)=\left(\alpha \eta\left(\alpha^{-1} x\right), \alpha \eta\left(\xi\left(\alpha^{-1} x\right)\right)\right.
$$

where $\alpha:=\alpha_{\xi, \eta}=[\eta(0)-\eta(\xi(0))]^{-1}$. Conditions $\left(c_{1}\right),\left(c_{2}\right)$ imply that $\alpha<-1$. The renormalization group transformation $\mathcal{R}$ has a single hyperbolic fixed point $\left(\xi_{0}, \eta_{0}\right)$ in the subspace $\mathcal{X}_{c r}(\bar{\rho})$ (see $[2,6,7]$.

Notice that $\xi_{0}(x), \eta_{0}(x)$ are real analytic functions of $x^{3}$ and the constant $\alpha_{0}:=\alpha_{\xi_{0}, \eta_{0}} \approx$ $\approx-1,2886$ (see $[2,6])$. Denote by $f_{c r}:=f_{\xi_{0}, \eta_{0}}$ the circle map associated with $\left(\xi_{0}, \eta_{0}\right)$.

Yoccoz in [8] generalized Denjoy's classical result and proved that a critical circle homeomorphism with an irrational rotation number is topologically conjugate to an irrational rotation.

Graczyk and Swiatek in [9] proved that if $f$ is $C^{3}$ circle homeomorphism with finitely many critical points of polynomial type and an irrational rotation number, then the conjugating map $\varphi$ is a singular function on $S^{1}$, i. e. $\varphi^{\prime}(x)=0$ a. e. on $S^{1}$. Consequently, the unique probability invariant measure $\mu_{f}$ of critical circle homeomorphisms $f$ is singular w.r.t. Lebesque measure on $S^{1}$.

The problem of smoothness of the conjugacy between two critical maps with identical irrational rotation number arises naturally. This is called the rigidity problem for critical circle homeomorphisms. For the critical circle maps the rigidity problem is developed by de Faria, de Melo, Yampolsky, Khanin and Teplinsky, Guarino among others.

Next we formulate the last fundamental result obtained by P. Guarino, M. Martens and W. de Melo in [10].

Theorem 1 (see [10]). Let $f_{1}$ and $f_{2}$ be two analytic $C^{4}$-circle homeomorphisms with the same irrational rotation number and with a unique critical point of the same odd type. Then they are $C^{1}$-smoothly conjugate to each other. The conjugacy is $C^{1+\epsilon}, \epsilon>0$, for Lebesgue almost every rotation number.

Denote by $\operatorname{Cr}(\bar{\rho})$ the set of all circle homeomorphisms, which are $C^{1}$-conjugated to $f_{c r}$ and defined on the standard circle $S^{1}=\mathbb{R} / \mathbb{Z} \simeq[0,1)$. It is well known (see [5]) that any two topological conjugated homeomorphisms have the same rotation number. Therefore, the rotation numbers of homeomorphisms of $C r(\bar{\rho})$ are the same and equal to $\bar{\rho}$.

Let $f$ be an orientation preserving a homeomorphism of the circle $S^{1}=\mathbb{R}^{1} / \mathbb{Z}^{1} \simeq[0,1)$ with an irrational rotation number $\rho=\rho_{f}$. Let $\mu=\mu_{f}$ be the unique invariant probability measure of $f$. Fix a point $z \in S^{1}$ and consider the interval $\mathfrak{J}_{\varepsilon}(z)=[z, z+\varepsilon] \subset S^{1}$. Consider the first hitting time of any $x \in[0,1)$ to the interval $\mathfrak{J}_{\varepsilon}(z)$ :

$$
N_{\varepsilon}^{(1)}(x)=\inf \left\{i \geq 1: f^{i}(x) \in \mathfrak{J}_{\varepsilon}(z)\right\} .
$$

The problem consists of finding conditions under which the hitting time, after rescaling by some suitable constant depending on $\mathfrak{J}_{\varepsilon}(z)$, converges in law, as $\mu\left(\mathfrak{J}_{\varepsilon}(z)\right)$ tends to zero. Since the expectation of the first hitting time is of the order $1 / \mu\left(\mathfrak{J}_{\varepsilon}(z)\right)$, it is natural to rescale the hitting time by this factor.

Next define the rescaled hitting time as

$$
E_{\varepsilon}^{(1)}(x)=\mu\left(\mathfrak{J}_{\varepsilon}(z)\right) N_{\varepsilon}^{(1)}(x) .
$$

We are interested in the convergence of the distribution function of the random variable $E_{\varepsilon}^{(1)}(x)$, i. e. in the convergence of the distribution function

$$
F_{\varepsilon}(t)=\mu\left(x \in S^{1}: E_{\varepsilon}^{(1)}(x) \leq t\right), \quad \forall t \in \mathbb{R}^{1},
$$


as $\varepsilon \rightarrow 0$, for every $t$ belonging to the continuity points of the limit function.

Coelho and de Faria in [11], Coelho in [12] investigated the problem of convergence of random variables $E_{\varepsilon}^{(1)}(t)$ for linear irrational rotations $f_{\rho}(x)=x+\rho \bmod 1$. It is known that for linear irrational rotation $f_{\rho}$ the Lebesgue measure $\ell$ is the unique invariant measure. In [11], $F_{n}(t)$ was studied when $\left[x_{0}, c_{n}\right]$ is $n$-th renormalization interval for map $f$. It is shown that for Lebesgue almost every rotation number $\rho$, the rescaled hitting times $E^{(1)}(\cdot):=\mu\left(\left[x_{c}, c_{n}\right]\right) N_{n}^{(1)}(\cdot)$ do not converge in law as $c_{n}$ tends to zero, and all possible limit laws under a subsequence of $\left\{c_{n}, n=1,2, \ldots\right\}$ are obtained.

Fix $\theta \in(0,1)$. Let $q_{n}, n \geq 1$, be the denominator of $n$-th convergent of continued fraction of an irrational $\bar{\rho}$ (for more details see Section 2). For every $n \geq 1$ we define the points $c_{n}(\theta)$ by:

$$
\mu\left(\left[x_{0}, c_{n}(\theta)\right]\right)=\theta \cdot \mu\left(\left[x_{0}, f^{q_{n}}\left(x_{0}\right)\right]\right) .
$$

Consider the hitting times $N_{n, \theta}^{(1)}, n \geq 1$, to the intervals $\left[x_{0}, c_{n}(\theta)\right)$ and rescaled hitting times $E_{n, \theta}^{(1)}(x):=\mu\left(\left[x_{0}, c_{n}(\theta)\right) N_{n, \theta}^{(1)}(x)\right.$. There are two natural measures on the circle: the invariant probability measure $\mu$ and the Lebesgue measure $\ell$. We define the distribution functions of $E_{n, \theta}^{(1)}(x)$ :

$$
\begin{array}{ll}
F_{\theta, n}(t)=\mu\left(x \in S^{1}: E_{n, \theta}^{(1)}(x) \leq t\right), & t \in \mathbb{R}^{1}, \\
\Phi_{\theta, n}(t)=\ell\left(x \in S^{1}: E_{n, \theta}^{(1)}(x) \leq t\right), & t \in \mathbb{R}^{1} .
\end{array}
$$

Z. Coelho in [12] investigated all the possible limit distributions for $F_{\theta, n}(t)$ under a subsequence $c_{n} \rightarrow 0$. It is shown that for every convergent subsequence $F_{\theta, n_{m}}(t), m=1,2, \ldots$, the limit distribution $F_{\theta}(t)$ is piecewise linear on $[0,1]: F_{\theta}(t)=0, t \leq 0$, and $F_{\theta}(t)=1, t \geq 1$. Notice that the results are valid for all circle diffeomorphisms which are $C^{1}$-conjugate to a rigid irrational rotation $f_{\rho}(x):=x+\rho \bmod 1$ (see $\left.[13,14]\right)$. They are also valid if one replaces the invariant measure $\mu$ by the Lebesgue measure.

We should mention that asymptotic time distributions have been obtained in a number of contexts, when studying hitting and return times of neighborhoods of generic points in the natural scale of the measure of the neighbourhoods (see equation (0.1)). For finite state Markov chains and Anosov diffeomorphisms [15], Axiom A diffeomorphisms [16], piecewise expanding maps of the interval [17] are all exponential of parameter one.

In this paper we investigate the rescaled hitting times for critical circle maps $f \in \operatorname{Cr}(\bar{\rho})$. We formulate the main result of our work.

Theorem 2. Let $\bar{\rho}=\frac{\sqrt{5}-1}{2}$ and let $f \in C r(\bar{\rho})$ be a critical circle map. Consider for $\theta \in(0,1)$ the sequence of distribution functions $\left\{\Phi_{n, \theta}(t)\right\}_{n=1}^{\infty}$ with respect to the Lebesgue measure on the circle corresponding to the first rescaled hitting times $E_{n, \theta}^{(1)}(x)$ to the interval $\left[x_{c}, c_{n}(\theta)\right]$. Then

1) for all $t \in \mathbb{R}^{1}$ there exists a finite limit

$$
\lim _{n \rightarrow \infty} \Phi_{n, \theta}(t)=\Phi_{\theta}(t)
$$

where $\Phi_{\theta}(t)=0$, if $t \leq 0$, and $\Phi_{\theta}(t)=1$, if $t>1$;

2) the limit function $\Phi_{\theta}(t)$ is a strictly increasing on $[0,1]$ and continuous distribution function on $\mathbb{R}^{1}$;

3) $\Phi_{\theta}(t)$ is singular on $[0,1]$, i.e. $\frac{\Phi_{\theta}(t)}{d t}=0$ a. e. with respect to the Lebesgue measure $\ell$ on the circle. 


\section{§1. The thermodynamic formalism for critical circle maps of $\operatorname{Cr}(\bar{\rho})$}

We consider the critical circle homeomorphism $f \in C r(\bar{\rho})$ with one critical point $x_{0}:=x_{c r}$ and the golden mean rotation number $\bar{\rho}=\frac{\sqrt{5}-1}{2}=[1,1, \ldots, 1, \ldots]$. For $n \geq 1$, we write $p_{n} / q_{n}=[1,1, \ldots, 1]$ the convergent of $\bar{\rho}$, their denominators $q_{n}$ satisfy the recursive relation $q_{n+1}=q_{n}+q_{n-1}$ with initial conditions $q_{0}=1, q_{1}=1$. The forward orbit $O_{f}^{+}\left(x_{0}\right)=$ $=\left\{x_{i}=f^{i}\left(x_{0}\right), i=0,1,2, \ldots\right\}$ of a critical point defines a sequence of natural partitions of the circle (see [5]). Indeed, denote by $I_{0}^{(n)}:=I_{0}^{(n)}\left(x_{0}\right)$ the closed interval in $S^{1}$ with the endpoints $x_{0}$ and $x_{q_{n}}=f^{q_{n}}\left(x_{0}\right)$. In the clockwise orientation of the circle, the point $x_{q_{n}}$ lies to the left of $x_{0}$ for odd $n$, and to the right for even $n$. If $I_{i}^{(n)}=f^{i}\left(I_{0}^{(n)}\right), i \geq 1$, denote the iterates of the interval $I_{0}^{(n)}$ under $f$, it is well known, that the set $\mathcal{P}_{n}:=\mathcal{P}_{n}\left(x_{0}\right)$ of intervals with mutually disjoint interiors, defined as

$$
\mathcal{P}_{n}=\left\{I_{i}^{(n)}, 0 \leq i<q_{n+1}\right\} \cup\left\{I_{j}^{(n+1)}, 0 \leq j<q_{n}\right\}
$$

determines a partition $\mathcal{P}_{n}$ of the circle for any $n$. The partition $\mathcal{P}_{n}$ is called the $n$-th dynamical partition of $S^{1}$ determined by the point $x_{0}$ and the map $f$. Note that, under the transition from $\mathcal{P}_{n}$ to $\mathcal{P}_{n+1}$, the intervals $I_{j}^{(n+1)}, 0 \leq j \leq q_{n}$, of rank $n+1$ are preserved, whereas the intervals $I_{i}^{(n)}$, $0 \leq i \leq q_{n+1}$, with rank $n$ are partitioned into two new intervals:

$$
I_{i}^{(n)}=I_{i}^{(n+2)} \cup I_{i+q_{n}}^{(n+1)} .
$$

One has the following lemma.

Lemma 1 (see [19]). Let $k>0$. Suppose $I^{(n)} \in \mathcal{P}_{n}\left(x_{0}\right), I^{(n-k)} \in \mathcal{P}_{n-k}\left(x_{0}\right), n>k$, and $I^{(n)} \subset I^{(n-k)}$. Then

$$
\frac{\left|I^{(n)}\right|}{\left|I^{(n-k)}\right|} \leq \text { Const }\left|\alpha_{0}\right|^{-k}, \quad \text { const }\left|\alpha_{0}\right|^{-3 n} \leq\left|I^{(n)}\right|
$$

Here and throughout on $|\cdot|$ denotes the length of an interval. The last estimates can be proved similarly to the assertion for Feigenbaum map [18]. The last estimates imply that the orbit of a critical point $x_{0} \in S^{1}$ is dense in $S^{1}$. This together with monotonicity of $f$ implies that the homeomorphism $f$ is topologically conjugate to the linear rotation $f_{\rho}(x)=x+\bar{\rho} \bmod 1$.

The sequence of dynamical partitions $\mathcal{P}_{n}$ allows us to introduce a symbolic dynamics for the map $f$. For this, take an arbitrary point $x \in S^{1} \backslash O_{f}^{+}$where $O_{f}^{+}$denotes the forward orbit of the critical point $x_{0}$ of $f$. For $n \geq 0$, put $a_{n+1}:=a_{n+1}(x)=a$ if $x \in I_{j}^{(n+1)}\left(x_{0}\right), 0 \leq j<q_{n}$. However, if $x \in I_{j}^{(n)}, 0 \leq j<q_{n+1}$, it follows from the construction of the partition $\mathcal{P}_{n+1}$ via $\mathcal{P}_{n}$ that in the case $\rho(f)=\omega=\frac{\sqrt{5}-1}{2}$ we have either $x \in I_{i}^{(n+2)}, 0 \leq i<q_{n+1}$, or $x \in I_{i+q_{n}}^{(n+1)}$, $0 \leq i<q_{n+1}$. In the first case we put $a_{n+1}=0$ and in the second one $a_{n+1}=1$. By this way we get a one-to-one correspondence

$$
\begin{gathered}
\varphi: S^{1} \backslash O_{f}^{+} \leftrightarrow\left\{\left(a_{0}, a_{1}, \ldots, a_{n}, \ldots, a_{n} \in\{a, 0,1\}\right.\right. \text { such that } \\
\left.a_{n+1}=0 \Longleftrightarrow a_{n}=a, \quad n \geq 1\right\}:=X_{+} .
\end{gathered}
$$

Notice that every interval $I^{(n)}$ of the dynamical partition $\mathcal{P}_{n}$ corresponds to the unique finite word $\left(a_{0}, a_{2}, \ldots, a_{n}\right)$ of length $n+1$. In particular, for $n$ odd, the words $(a, 0, a, 0, \ldots, a, 0)$ and $(0, a, 0, a, \ldots, 0, a)$ correspond to the intervals $I_{0}^{(n)}$ and $I_{0}^{(n+1)}$, respectively. Next, we define another space of one-sided infinite words with the same alphabet $\mathbf{A}=\{a, 0,1\}$.

$$
Y_{+}:=\left\{\underline{a}=\left(a_{1}, a_{2}, \ldots, a_{n}, \ldots\right), a_{n} \in \mathbf{A} \text {, such that } a_{n+1}=0 \Longleftrightarrow a_{n}=a, n \geq 1\right\} .
$$


Recall that $\alpha_{0}$ is the parameter of the scaling transformation of the corresponding pair $\xi_{0}, \eta_{0}$, i. e., $\alpha_{0}=\left[\eta_{0}(0)-\xi_{0}\right]^{-1}, \alpha_{0} \approx-1,2886 \ldots$ (see [2,3]). Since $x_{0}$ is a critical point of the map $f$, we can consider the neighborhood of the point $x_{1}: V\left(x_{1}\right):=I^{(1)}\left(x_{1}\right) \cup I^{(2)}\left(x_{1}\right)$.

We formulate the theorem on thermodynamical formalism for the critical circle maps from $\operatorname{Cr}(\bar{\rho})$.

Theorem 3 (see [19]). For $f \in C r(\bar{\rho})$, there is a unique, continuous (in the Tikhonov topology) function $U_{c r}: Y_{+} \rightarrow(-\infty, 0)$ with the following properties.

(1) Let $\underline{a}, \underline{b} \in Y_{+}, m \in Z_{+}$, and $a_{i}=b_{i}$ for all $1 \leq i \leq m$. Then

$$
\left|U_{c r}(\underline{a})-U_{c r}(\underline{b})\right| \leq C_{3} \cdot\left|\alpha_{0}\right|^{-m}
$$

where the constant $C_{3}>0$ does not depend on $\underline{a}, \underline{b}$ and $m$.

(2) Let $I_{s_{n}}^{(n)}, I_{s_{r}}^{(r)} \in \mathcal{P}_{n}, I_{s_{n}}^{(n)} \subset I_{s_{r}}^{(r)} \subset V\left(x_{1}\right), 1 \leq r<n$, and $\varphi\left(I_{s_{n}}^{(n)}\right)=\left(b_{1}, b_{2}, \ldots, b_{n}\right)$, $\varphi\left(I_{s_{r}}^{(r)}\right)=\left(b_{1}, b_{2}, \ldots, b_{r}\right)$. Then

$$
\left|I_{s_{n}}^{(n)}\right|=\left(1+\psi\left(b_{1}, b_{2}, \ldots, b_{n}\right)\right)\left|I_{s_{r}}^{(r)}\right| \exp \left\{\sum_{s=r}^{n} U_{c r}\left(b_{s}, \ldots, b_{1}, b_{r}, \ldots, b_{1}, \underline{\gamma}\left(b_{1}\right)\right)\right\},
$$

where $\left|\psi\left(b_{1}, b_{2}, \ldots, b_{n}\right)\right| \leq$ Const $\cdot\left|\alpha_{0}\right|^{-r}$, and

$$
\underline{\gamma}\left(b_{1}\right):= \begin{cases}(a, 0, a, 0, \ldots, a, 0, \ldots), & \text { if } b_{1}=0 \\ (0, a, 0, a, \ldots, 0, a, \ldots), & \text { if } b_{1}=a, 1 .\end{cases}
$$

Notice that the potential $U_{c r}$ is uniquely determined by the orbit of a critical point. The last assertion of the Theorem 3 implies that the length of the interval $I^{(n)} \in \mathcal{P}_{n}$ can be estimated as

$$
\text { const } \leq \frac{\left|I^{(n)}\right|}{\exp \left\{\sum_{s=1}^{n} U_{c r}\left(b_{s}, b_{s-1}, \ldots, b_{1}, \underline{\gamma}\left(b_{1}\right)\right)\right\}} \leq \text { Const. }
$$

Following the statistical mechanics terminology we call the function $U_{c r}:=U_{c r}\left(a_{1}, a_{2}, \ldots, a_{n}, \ldots\right)$ the potential corresponding to the critical maps $f \in C r(\omega)$ [20]. The first assertion of the Theorem 3 shows that the potential $U_{c r}\left(a_{1}, a_{2}, \ldots, a_{n}, \ldots\right)$ depends exponentially weakly on distant variables.

Fix an integer $k>0$. Define the $(n-k)$-th renormalization interval of the critical point $x_{0}$ by

$$
V_{n-k}:=\left[x_{q_{n-k+1}}, x_{q_{n-k}}\right]=I_{0}^{(n-k+1)} \cup I_{0}^{(n-k)} .
$$

We now consider the dynamical partitions $\mathcal{P}_{n-k}$ and $\mathcal{P}_{n}, n>k$, of the critical point $x_{0}$. We consider an arbitrary interval $J^{(n)}=\left(y^{(n)}, z^{(n)}\right), n>k$, lying, either in the interval $I_{0}^{(n-k)}$, or $I_{0}^{(n-k+1)}$. Notice that if $x, y \in f^{i}\left(I_{0}^{(n-k)}\right), 0 \leq i \leq q_{n-k}$, then in their symbolic representations first $n-k$ letters coincide.

Let the infinite words

$$
\left(b_{1}(0), b_{2}(0), \ldots, b_{n-k}(0), \varepsilon^{(1)}, \varepsilon^{(2)}, \ldots, \varepsilon^{(p)}, \ldots\right)
$$

and

$$
\left(b_{1}(0), b_{2}(0), \ldots, b_{n-k}(0), \gamma^{(1)}, \gamma^{(2)}, \ldots, \gamma^{(p)}, \ldots\right)
$$


correspond to the endpoints $y^{(n)}$ and $z^{(n)}$ of the interval $J^{(n)}$, respectively. Then due to $f^{i}\left(J^{(n)}\right) \subset$ $\subset f^{i}\left(I_{0}^{(n-k)}\right), 0 \leq i \leq q_{n-k}$, the words

$$
\left(b_{1}(i), b_{2}(i), \ldots, b_{n-k}(i), \varepsilon^{(1)}, \varepsilon^{(2)}, \ldots, \varepsilon^{(p)}, \ldots\right)
$$

and

$$
\left(b_{1}(i), b_{2}(i), \ldots, b_{n-k}(i), \gamma^{(1)}, \gamma^{(2)}, \ldots, \gamma^{(p)}, \ldots\right)
$$

correspond to the endpoints $f^{i}\left(y^{(n)}\right)$ and $f^{i}\left(z^{(n)}\right)$, respectively.

We set

$$
\begin{gathered}
\underline{\varepsilon}:=\left(\epsilon^{(1)}, \epsilon^{(2)}, \ldots, \epsilon^{(p)}, \ldots\right), \quad \underline{\gamma}:=\left(\gamma^{(1)}, \gamma^{(2)}, \ldots, \gamma^{(p)}, \ldots\right), \\
f^{i}\left(J^{n}\right):=J\left(b_{1}(i), b_{2}(i), \ldots, b_{n-k}(i) ; \underline{\varepsilon}, \underline{\gamma}\right) .
\end{gathered}
$$

Let the interval $J^{(n)}$ be $q_{n}$-small, i. e., the intervals $J^{(n)}, f\left(J^{(n)}\right), \ldots, f^{q_{n}-1}\left(J^{(n)}\right)$ are mutually disjoint (except their endpoints). We recall that $N_{n-k}^{(1)}(x)$ is the first hitting time function of the point $x$ to the interval $V_{n-k}$. The function $N_{n-k}^{(1)}(x)$ is a step function taking constant value on each interval $I^{(n-k)}$ of the partition $\mathcal{P}_{n-k}$.

For each $\beta>0$ we define the following sum:

$$
\left.S_{n-k}(\beta, \underline{\varepsilon}, \underline{\gamma}):=\sum_{i=0}^{N_{n-k}^{(1)}-1} \mid f^{i}\left(J^{n}\right)\right)\left.\right|^{\beta}=\sum_{i=0}^{N_{n-k}^{(1)}-1}\left|J\left(b_{1}(i), b_{2}(i), \ldots, b_{n-k}(i) ; \underline{\varepsilon}, \underline{\gamma}\right)\right|^{\beta} .
$$

Definition 1. Let $k>0$. The sequence of sums $\left\{S_{n-k}(\beta, \underline{\varepsilon}, \underline{\gamma}), n \geq k+1\right\}$ is called the sequence of $q_{n-k}$-sums of the map $f$ associated with infinite symbols $\underline{\varepsilon}$ and $\underline{\gamma}$.

We now formulate the theorem proved in [19] which will be useful in the proof of Theorem 2.

Theorem 4 (see [19]). For each $k>0, \beta>0, \underline{\varepsilon}$ and $\gamma$, there exists a finite limit

$$
\lim _{n \rightarrow \infty} S_{n-k}(\beta, \underline{\varepsilon}, \underline{\gamma})=r_{k}(\beta, \underline{\varepsilon}, \underline{\gamma})
$$

The following theorem gives the lower and upper bounds for $q_{n-k}$-sums $S_{n-k}(\beta, \underline{\varepsilon}, \underline{\gamma})$ and their limit $r_{k}(\beta, \underline{\varepsilon}, \underline{\gamma})$.

Theorem 5. For each $n \geq k+1$, the following estimates

$$
C_{4}^{-1} \cdot\left|J^{(n)}\right| \leq S_{n-k}(\beta, \underline{\varepsilon}, \underline{\gamma}) \leq C_{4} \cdot\left|J^{(n)}\right|
$$

hold, where the constant $C_{4}>1$ doesn't depend on $n, k, \underline{\varepsilon}$ and $\underline{\gamma}$.

P r o o f. First we prove the statement of the Theorem 5 in the case when $J^{(n)}$ is an interval of the dynamical partition $\mathcal{P}_{n}$. We consider the homeomorphism $f_{\xi_{0}, \eta_{0}}$ of the circle $\left[\eta_{0}(0), \xi_{0}(0)\right)$, which corresponds to the pair of functions $\left(\xi_{0}(x), \eta_{0}(x)\right)$. We recall that $\left(\xi_{0}(x), \eta_{0}(x)\right)$ is a fixed point of the renormalization group transformation $\mathcal{R}$. We denote by $\tau_{n}$ the $n$-th dynamical partition of $f_{\xi_{0}, \eta_{0}}$ and its intervals by $\Delta$. We have

$$
\tau_{n}:=\left\{\Delta_{i}^{n}, 0 \leq i<q_{n+1} ; \Delta_{j}^{n+1}, 0 \leq j<q_{n}\right\} .
$$

By definition, the homeomorphism $f \in C r(\bar{\rho})$ is $C^{1}$-conjugate to $f_{\xi_{0}, \eta_{0}}$, i.e., there exists a diffeomorphism $\psi \in C^{1}\left(S^{1}\right)$ such that $\psi \circ f=f_{\xi_{0}, \eta_{0}} \circ \psi$. Hence, the intervals $I^{n} \in \mathcal{P}$ and 
$\Delta^{n}=\psi\left(I^{n}\right) \in \tau_{n}$ are comparable. Therefore we can prove the statement of the lemma for the homeomorphism $f_{\xi_{0}, \eta_{0}}$. We set $J^{(n)}:=\Delta^{(n)} \in \tau_{n}$. To be definite, suppose $\Delta^{(n)} \subset \Delta^{(n-k+1)} \subset$ $\subset \Delta_{0}^{(1)}$.

First, we estimate the following sum

$$
S_{n-k}(\beta)=\sum_{b_{1}, b_{2}, \ldots, b_{n-k+1}}\left|\Delta\left(b_{1}, b_{2}, \ldots, b_{n-k+1}, a, 0, \ldots, a, 0\right)\right|^{\beta},
$$

where the sum ranges over all admissible $b_{1}, b_{2}, \ldots, b_{n-k+1}$.

We rewrite the lengths of intervals in the last sum using the potential $U_{c r}$. Note that the thermodynamic formalism for $f_{\xi_{0}, \eta_{0}}$ was constructed for intervals lying in the neighborhood of $x_{1}: V_{1}\left(x_{1}\right)=\Delta_{0}^{(1)}\left(x_{1}\right) \cup \Delta_{0}^{(2)}\left(x_{1}\right)$. It follows from the definition of symbolic dynamics that

$$
\begin{gathered}
\Delta^{(n)}:=\Delta^{(n)}\left(a, 1,0, b_{4}, b_{5}, \ldots, b_{n-k-1}, \varepsilon^{(k)}, \ldots, \varepsilon^{(2)}, \varepsilon^{(1)}\right), \text { if } \Delta^{(n)} \subset \Delta_{0}^{(2)}\left(x_{1}\right), \\
\Delta^{n}:=\Delta^{n}\left(1,0, a, a_{4}, a_{5}, \ldots, a_{n-k-1}, \varepsilon^{(k)}, \ldots, \varepsilon^{(2)}, \varepsilon^{(1)}\right), \text { if } \Delta^{(n)} \cup \Delta_{0}^{(2)}\left(x_{1}\right) .
\end{gathered}
$$

Using Lemma 1 , the sum $S_{n-k}(\beta, \underline{\varepsilon}, \underline{\gamma})$ can be written in the form

$$
\begin{aligned}
S_{n-k}(\beta, \underline{\varepsilon}, \underline{\gamma})= & \sum_{s=0}^{n-k-4}\left|\alpha_{0}\right|^{-s}\left\{\sum_{b_{4}, b_{5}, \ldots, b_{n-k-1}}\left|\Delta\left(a, 1,0, b_{4}, b_{5}, \ldots, b_{n-k-1}, \varepsilon^{(k)}, \ldots, \varepsilon^{(2)}, \varepsilon^{(1)}\right)\right|+\right. \\
& \left.+\sum_{a_{4}, a_{5}, \ldots, a_{n-k-1}}\left|\Delta\left(a, 1,0, a_{4}, a_{5}, \ldots, a_{n-k-1}, \varepsilon^{(k)}, \ldots, \varepsilon^{(2)}, \varepsilon^{(1)}\right)\right|\right\},
\end{aligned}
$$

where the constant $\alpha_{0} \simeq-1,28$ [2]. Theorem 3 implies that

$$
\begin{aligned}
& \Delta\left(x_{1}, x_{2}, \ldots,\right.\left.x_{n-k-s-1}, \varepsilon^{(k)}, \ldots, \varepsilon^{(2)}, \varepsilon^{(1)}\right)=\left|\Delta\left(x_{1}, x_{2}, \ldots, x_{n-k-s-1}\right)\right| \times \\
& \times\left(1+\psi_{1}\left(x_{1}, x_{2}, \ldots, x_{n-k-s-1}, \varepsilon^{(k)}, \ldots, \varepsilon^{(2)}, \varepsilon^{(1)}\right) \times\right. \\
& \exp \left\{\sum_{l=0}^{k-1} U_{c r}\left(\varepsilon^{(k-l)}, \ldots, \varepsilon^{(k)}, x_{n-k-s-1}, \ldots, x_{1}, \underline{\gamma}\left(x_{1}\right)\right)\right\},
\end{aligned}
$$

where $\left|\psi_{1}\right| \leq$ const $\cdot\left|\alpha_{0}\right|^{-(n-k-s-1)}$. Let $\Delta\left(x_{1}, x_{2}, \ldots, x_{n-k-s-1}, \varepsilon^{(k)}, \ldots, \varepsilon^{(2)}, \varepsilon^{(1)}\right) \subset V_{1}$. Using the estimates (1.1), we obtain:

$$
\begin{gathered}
C_{2} \cdot \exp \left\{\sum_{l=0}^{k-1} U_{c r}\left(\varepsilon^{(k-l)}, \ldots, \varepsilon^{(k)}, x_{n-k-s-1}, \ldots, x_{1}, \underline{\gamma}\left(x_{1}\right)\right)\right\} \leq\left|\Delta\left(\varepsilon^{(k)}, \ldots, \varepsilon^{(2)}, \varepsilon^{(1)}\right)\right| \leq \\
C_{3} \cdot \exp \left\{\sum_{l=0}^{k-1} U_{c r}\left(\varepsilon^{(k-l)}, \ldots, \varepsilon^{(k)}, x_{n-k-s-1}, \ldots, x_{1}, \underline{\gamma}\left(x_{1}\right)\right)\right\} .
\end{gathered}
$$

Using the last estimates, (1.2) and (1.3), we find that

$$
C_{4} \leq \frac{S_{n-k}(\beta, \underline{\varepsilon}, \underline{\gamma})}{\left|\Delta\left(\varepsilon^{(k)}, \ldots, \varepsilon^{(1)}\right)\right|} \leq C_{5}
$$

Now we prove the statement of Theorem 5 in the case where $J^{(n)}$ is $q_{n}$-small but not an interval of dynamical partition $\mathcal{P}_{n}\left(x_{0}\right)$. We consider the dynamical partition $\mathcal{P}_{n+m}\left(x_{0}\right), m \geq 1$. Denote by $\omega_{1}, \omega_{2}, \ldots, \omega_{l(m)}$ the intervals of the partition $\mathcal{P}_{n+m}\left(x_{0}\right), m \geq 1$, whose union covers $J^{(n)}$. Because $J^{(n)}$ is a $q_{n}$-small interval, it follows from the dynamical partition structure that 
$l(m) \leq$ const $\cdot q_{m+1}$. The last two statements of Theorem 3 imply that for each $\omega \in \mathcal{P}_{n+m}\left(x_{0}\right)$, we have

$$
\text { const } \cdot d_{1}^{n+n} \leq|\omega| \leq \text { Const } \cdot d_{2}^{n+m} \text {, }
$$

where the constants $0<d_{1}<d_{2}<1$ don't depend on $n$ and $m$. Hence, for sufficiently large $m$,

$$
\bigcup_{s=1}^{l(m)} \omega_{s} \subset V_{n-k}
$$

It is clear that

$$
f^{i}\left(J^{(n)}\right) \subset \bigcup_{s=1}^{l(m)} f^{i}\left(\omega_{s}\right), \quad i \geq 0
$$

We set $N_{n-k}^{(1)}:=\pi_{n-k}\left(I^{(n)}\right)$. Using the second inequality of Lemma 1, we can show that

$$
1 \leq\left|f^{i}\left(J^{(n)}\right)\right| \sum_{j=0}^{l(m)}\left|f^{i}\left(\omega_{j}\right)\right| \leq 1+\text { const } \cdot d_{2}^{m}, \quad 0 \leq i \leq N_{n-k}^{(1)}-1 .
$$

The last inequalities imply that

$$
1 \leq\left\{\sum_{j=0}^{N_{n-k}^{(1)}-1} \sum_{j=1}^{l(m)}\left|f^{i}\left(\omega_{j}\right)\right|\right\} /\left\{\sum_{i=0}^{N_{n-k}^{(1)}-1}\left|f^{i}\left(J^{(n)}\right)\right|\right\} \leq 1+\text { const } \cdot d_{2}^{m} .
$$

The last relations together with (1.4) imply the assertion of Theorem 5 for the interval $J^{n}$.

\section{$\S 2$. Hitting times of circle maps}

We consider the linear rotation $f_{\rho}(x)=x+\rho \bmod 1, x \in[0,1)$, to the irrational angle $\rho \in(0,1)$. Let $A \subset \mathbb{R}^{1}$ be a measurable subset with $\ell(A)>0$. Define the first return time $R_{A}: A \rightarrow \mathbb{N}$ as

$$
R_{A}(x)=\inf \left\{i \geq 1: f_{\rho}^{i}(x) \in A\right\} \text { for all } x \in A
$$

For $t \in \mathbb{R}^{1}$ we define by

$$
\|t\|=\min _{n \in \mathbb{Z}}|t-n|
$$

the distance to the nearest integer. It is known that the first return time $R_{A}$ of an irrational rotation $f_{\rho}(x)$ has at most three values if $A$ is an interval (see [13]).

Proposition 1 (see [13]). Let $f_{\rho}$ be an irrational rotation and $b \in(0,\|\rho\|)$ be a fixed number. Let $n=n_{b} \geq 0$ be an integer such that $\left\|q_{n+1} \rho\right\|<b \leq\left\|q_{n} \rho\right\|$ and $K:=K_{b}$ an integer, which satisfies

$$
K=\max \left\{k \geq 0: k\left\|q_{n+1} \rho\right\|+\left\|q_{n+2} \rho\right\|<b\right\} .
$$

If $n \geq 0$ is odd, then

$$
R_{[0, b)}(x)= \begin{cases}q_{n+1}, & x \in\left[0, b-\left\|q_{n+1} \rho\right\|\right), \\ q_{n+2}-(K-1) q_{n+1}, & x \in\left[b-\left\|q_{n+1} \rho\right\|, K\left\|q_{n+1} \rho\right\|+\left\|q_{n+2} \rho\right\|\right), \\ q_{n+2}-K q_{n+1}, & x \in\left[K\left\|q_{n+1} \rho\right\|+\left\|q_{n+2} \rho\right\|, b\right) .\end{cases}
$$

If $n \geq 0$ is even, then

$$
R_{[0, b)}(x)= \begin{cases}q_{n+2}-K q_{n+1}, & x \in\left[0, b-K\left\|q_{n+1} \rho\right\|-\left\|q_{n+2} \rho\right\|\right), \\ q_{n+2}-(K-1) q_{n+1}, & x \in\left[b-K\left\|q_{n+1} \rho\right\|-\left\|q_{n+2} \rho\right\|,\left\|q_{n+1} \rho\right\|\right), \\ q_{n+1}, & x \in\left[\left\|q_{n+1} \rho\right\|, b\right) .\end{cases}
$$


Remark 1. Note that the value $R_{[0, b)}(x)$ at the middle interval is the sum of two other values.

Remark 2. $0 \leq K \leq k_{i+1}-1$ for all $i \geq 0$.

Let $f$ be a circle homeomorphism with the golden mean rotation number $\alpha$ and with a unique probability invariant measure $\mu:=\mu_{f}$. Take an arbitrary point $x_{0} \in S^{1}$.

Take an arbitrary point $c_{1} \in\left[f^{q_{1}}\left(x_{0}\right), 1\right)$. There exists a constant $\theta \in(0,1]$ such that

$$
\mu\left(\left[x_{0}, c_{1}\right]\right):=\theta \cdot \mu\left(\left[f^{q_{1}}\left(x_{0}\right), 1\right]\right):=\theta \cdot \mu\left(I_{0}^{(1)}\right) .
$$

For every $n \geq 1$ we define $c_{n:}=c_{n}(\theta) \in I_{0}^{(n)}\left(x_{0}\right)$ as

$$
\mu\left(I_{c_{n}}\left(x_{0}\right)\right):=\theta \cdot \mu\left(I_{0}^{(n)}\left(x_{0}\right)\right),
$$

where the interval $I_{c_{n}}\left(x_{0}\right)$ has endpoints $x_{0}$ and $c_{n}$. It is easy to check that $c_{n}$ lies between $f^{-q_{n+1}}\left(x_{0}\right)$ and $f^{q_{n}}\left(x_{0}\right)$ for all $n \geq 1$.

Consider the first return time function:

$$
R_{c_{n}}(x)=\min \left\{j \geq 1: f^{j}(x) \in I_{c_{n}}\right\} .
$$

The Remark 1 implies that the golden mean rotation number $K$ defined in Proposition 1 equals to zero, i.e., $K:=K_{c_{n}}=0$ for all $n \geq 2$. Applying the Proposition 1 we obtain the following useful fact.

Proposition 2. Let $f$ be a circle homeomorphism with the golden mean rotation number $\alpha$ and $x_{0} \in S^{1}$. Assume the constant $\theta \in(0,1)$ and $c_{n} \in I_{0}^{(n)}\left(x_{0}\right), n>1$, are determined by (2.1) and (2.2), respectively.

(I) If $n \in \mathbb{N}$ is odd, then

$$
R_{c_{n}}(x)= \begin{cases}q_{n+2}, & x \in\left[c_{n}, f^{q_{n+2}}\left(x_{0}\right)\right) \\ q_{n+3}, & x \in\left[f^{q_{n+2}}\left(x_{0}\right), f^{q_{n+1}}\left(c_{n}\right)\right), \\ q_{n+1}, & x \in\left[f^{-q_{n+1}}\left(c_{n}\right), x_{0}\right) .\end{cases}
$$

(II) If $n \in \mathbb{N}$ is even, then

$$
R_{c_{n}}(x)= \begin{cases}q_{n+2}, & x \in\left[x_{0}, f^{-q_{n+2}}\left(c_{n}\right)\right), \\ q_{n+3}, & x \in\left[f^{-q_{n+2}}\left(c_{n}\right), f^{q_{n+1}}\left(x_{0}\right)\right), \\ q_{n+1}, & x \in\left[f^{q_{n+1}}\left(x_{0}\right), c_{n}\right) .\end{cases}
$$

To be definite, we consider the case when $n$ is even. The case of odd $n$ can be considered similarly. Introduce the following notations:

$$
A_{0}^{(n)}=\left[x_{0}, f^{-q_{n+2}}\left(c_{n}\right)\right), \quad C_{0}^{(n)}=\left[f^{-q_{n+2}}\left(c_{n}\right), f^{q_{n+1}}\left(x_{0}\right)\right), \quad B_{0}^{(n)}=\left[f^{q_{n+1}}\left(x_{0}\right), c_{n}\right) .
$$

The collection of intervals

$$
\begin{gathered}
\xi_{n}\left(x_{0}, c_{n}\right)=\left\{A_{0}^{(n)}, f\left(A_{0}^{(n)}\right), \ldots, f^{q_{n+2}}\left(A_{0}^{(n)}\right)\right\} \cup\left\{C_{0}^{(n)}, f\left(C_{0}^{(n)}\right), \ldots, f^{q_{n+3}}\left(C_{0}^{(n)}\right)\right\} \cup \\
\cup\left\{B_{0}^{(n)}, f\left(B_{0}^{(n)}\right), \ldots, f^{q_{n+1}}\left(B_{0}^{(n)}\right)\right\}
\end{gathered}
$$

constitutes the partition of the circle $S^{1}$. We denote it by $\xi_{n}\left(x_{0}, c_{n}\right)$ and call the $n$-th generalized dynamical partition associated with the points $x_{0}$ and $c_{n}$.

Using the properties of dynamical partitions, one can show the following proposition. 
Proposition 3. The location of intervals of the generalized dynamical partition $\xi_{n}\left(x_{0}, c_{n}\right)$ in intervals of dynamical partition $\mathcal{P}\left(x_{0}\right)$ is following (see Fig 4.1, 4.2):

1)

$$
f^{i}\left(A_{0}^{(n)} \cup C_{0}^{(n)} \cup B_{0}^{(n)}\right) \subset I_{i}^{(n)}, \quad 0 \leq i \leq q_{n+1}-1 ;
$$

2)

$$
\begin{gathered}
f^{i}\left(A_{0}^{(n)} \cup C_{0}^{(n)}\right)=I_{i}^{(n+1)}, \quad q_{n+1} \leq i \leq q_{n+2}-1 ; \\
f^{q_{n+1}}\left(B_{0}^{(n)}\right)=\left[x_{0}, c_{n+q_{n+2}}\right) \subset I_{0}^{(n+2)} ;
\end{gathered}
$$

3)

$$
\begin{gathered}
f^{i}\left(C_{0}^{(n)}\right)=f^{i-q_{n+2}}\left(\left[c_{n}, x_{q_{n}}\right)\right) \subset f^{i-q_{n+2}}\left(I_{0}^{(n)}\right), \quad q_{n+2} \leq i \leq q_{n+3}-1, \\
f^{q_{n+2}}\left(A_{0}^{(n)}\right)=\left[x_{q_{n+2}}, c_{n}\right) \subset I_{0}^{(n)} .
\end{gathered}
$$

Recall that, the first hitting time function in $S^{1}$ is

$$
N_{n}^{(1)}(x)=\min \left\{j \geq 1: f^{j}(x) \in I_{c_{n}}\right\}, \quad x \in S^{1} .
$$

Proposition 4. Hitting time function is given by

$$
N_{n}^{(m)}(x)=\left\{\begin{array}{lll}
m q_{n+1}-i, & \text { if } x \in f^{i}\left(B_{0}^{(n)}\right), & 1 \leq i \leq q_{n+1}, \\
m q_{n+2}-j, & \text { if } x \in f^{j}\left(A_{0}^{(n)}\right), & 1 \leq j \leq q_{n+2}, \\
m q_{n+3}-k, & \text { if } x \in f^{k}\left(C_{0}^{(n)}\right), & 1 \leq k \leq q_{n+3} .
\end{array}\right.
$$

P r o o f. Note that the collection of intervals

$$
A_{i}^{(n)}, \quad 0 \leq i<q_{n+2}, \quad B_{j}^{(n)}, \quad 0 \leq j<q_{n+1}, \quad C_{k}^{(n)}, \quad 0 \leq k<q_{n+3},
$$

is a partition of the circle. This implies the required assertion of Proposition.

Now we rescale the hitting time function $N_{n}^{(1)}(x)$, i. e., we divide it by the largest value and denote by $E_{n}^{(1)}(x)$, i. e.,

$$
E_{n}^{(1)}(x)=\frac{1}{q_{n+3}} N_{n}^{(1)}(x) .
$$

The last proposition implies that $N_{n}^{(1)}(x)$ gets values from 1 to $q_{n+3}$. Obviously, the rescaled function $E_{n}^{(1)}(x)$ is a random variable taking values in $(0,1]$. We denote by $\Phi_{n, \theta}(t)$ the distribution function of $E_{n}^{(1)}(x)$ w. r. t. Lebesque measure on $S^{1}$.

We formulate the following theorem.

Theorem 6. The distribution function of the rescaled hitting time function $E_{n}^{(1)}(x)$ has the following form:

i) if $t<1 / q_{n+3}$, then $\Phi_{n, \theta}(t)=0$;

ii) if $m / q_{n+3} \leq t \leq(m+1) / q_{n+3}, 1 \leq m \leq q_{n+1}$, then

$$
\Phi_{n, \theta}(t)=\sum_{i=q_{n+1}-m}^{q_{n+1}-1}\left|B_{i}^{(n)}\right|+\sum_{j=q_{n+2}-m}^{q_{n+2}-1}\left|A_{j}^{(n)}\right|+\sum_{k=q_{n+3}-m}^{q_{n+3}-1}\left|C_{k}^{(n)}\right| ;
$$


iii) if $m / q_{n+3} \leq t \leq(m+1) / q_{n+3}, q_{n+1} \leq m \leq q_{n+2}$, then

$$
\Phi_{n, \theta}(t)=\sum_{i=0}^{q_{n+1}-1}\left|B_{i}^{(n)}\right|+\sum_{j=q_{n+2}-m}^{q_{n+2}-1}\left|A_{j}^{(n)}\right|+\sum_{k=q_{n+3}-m}^{q_{n+3}-1}\left|C_{k}^{(n)}\right|
$$

iv) if $m / q_{n+3} \leq t \leq(m+1) / q_{n+3}, q_{n+2} \leq m \leq q_{n+3}$, then

$$
\Phi_{n, \theta}(t)=\sum_{i=0}^{q_{n+1}-1}\left|B_{i}^{(n)}\right|+\sum_{j=0}^{q_{n+2}-1}\left|A_{j}^{(n)}\right|+\sum_{k=q_{n+3}-m}^{q_{n+3}-1}\left|C_{k}^{(n)}\right| ;
$$

v) if $t \geq 1$, then $\Phi_{n, \theta}(t)=1$, where $\left|L_{i}^{(n)}\right|$ is Lebesque measure of $L_{i}^{n}$.

P r o o f. Since all elements of the $n$-th dynamical partition $\mathcal{P}_{n}$ fully cover the unit circle, any point $x \in S^{1}$ belongs to either $I_{i}^{n}$ or $I_{j}^{n+1}$ for $0 \leq i<q_{n+1}$ and $0 \leq j<q_{n}$. Using Proposition 3, we have that $x \in S^{1}$ belongs either to $A_{i}^{(n)}, 0 \leq i<q_{n+2}$, or $B_{j}^{(n)}, 0 \leq j<q_{n+1}$, or $C_{k}^{(n)}$, $0 \leq k<q_{n+3}$. If $m / q_{n+3} \leq t \leq(m+1) / q_{n+3}$ and $1 \leq m \leq q_{n+1}$, then the following equality holds

$$
\ell\left(x \in S^{1}: E_{n}^{(1)}(x)=t\right)=\left|B_{i}^{(n)}\right|+\left|A_{j}^{(n)}\right|+\left|C_{k}^{(n)}\right| .
$$

For $q_{n+1} \leq m \leq q_{n+2}$,

$$
\ell\left(x \in S^{1}: E_{n}^{(1)}(x)=t\right)=\left|A_{j}^{(n)}\right|+\left|C_{k}^{(n)}\right| .
$$

And for $q_{n+2} \leq m \leq q_{n+3}$, we have

$$
\ell\left(x \in S^{1}: E_{n}^{(1)}(x)=t\right)=\left|C_{k}^{(n)}\right| .
$$

Using Proposition 4 we get the assertions in ii), iii), iv). Therefore, since $\Phi_{n, \theta}(t)$ is a step function, it is clear that $\Phi_{n, \theta}(t)=0$ if $t \leq 0$, and $\Phi_{n, \theta}(t)=1$ if $t \geq 1$. Theorem 6 is proved.

Next we introduce the following distribution function:

$$
\Psi_{n, \theta}(t)=\left\{\begin{array}{l}
0, \text { if } t \leq 0, \\
\sum_{k=0}^{l}\left|C_{k}^{(n)}\right|, \text { if } l q_{n+3}^{-1} \leq t \leq(l+1) q_{n+3}^{-1}, 1 \leq l \leq q_{n+1}, \\
\sum_{i=0}^{l-q_{n+1}}\left|A_{i}^{(n)}\right|+\sum_{k=0}^{l}\left|C_{k}^{(n)}\right|, \text { if } l q_{n+3}^{-1} \leq t \leq(l+1) q_{n+3}^{-1}, q_{n+1} \leq l<q_{n+2}, \\
\sum_{j=0}^{l-q_{n+2}}\left|B_{j}^{(n)}\right|+\sum_{i=0}^{l-q_{n+1}-1}\left|A_{i}^{(n)}\right|+\sum_{k=0}^{l}\left|C_{k}^{(n)}\right|, \text { if } l q_{n+3}^{-1} \leq t \leq(l+1) q_{n+3}^{-1}, q_{n+2} \leq l<q_{n+3}, \\
1, \text { if } t \geq 1,
\end{array}\right.
$$

where $l=q_{n+3}-m-1$.

One has the following proposition.

Proposition 5. For all $n \geq 1$ the following relation holds:

$$
\Phi_{n, \theta}(t)=1-\Psi_{n, \theta}(1-t), \quad t \in \mathbb{R}^{1} .
$$


P r o o f. In view of Theorem 6 and structure of dynamical partitions, we present $\Phi_{n, \theta}(t)$ in a convinient form. Let $m q_{n+3}^{-1} \leq t \leq(m+1) q_{n+3}^{-1}, q_{n+2} \leq m \leq q_{n+3}$. We have

$$
\Phi_{n, \theta}(t)=\sum_{i=0}^{q_{n+1}-1}\left|B_{i}^{(n)}\right|+\sum_{j=0}^{q_{n+2}-1}\left|A_{j}^{(n)}\right|+\sum_{k=q_{n+3}-m}^{q_{n+3}-1}\left|C_{k}^{(n)}\right| .
$$

Hence

$$
\begin{aligned}
\Psi_{n, \theta}(1-t)=1-\Phi_{n, \theta}(t)=1- & \left\{\sum_{i=0}^{q_{n+1}-1}\left|B_{i}^{(n)}\right|+\sum_{j=0}^{q_{n+2}-1}\left|A_{j}^{(n)}\right|+\sum_{k=q_{n+3}-m}^{q_{n+3}-1}\left|C_{k}^{(n)}\right|\right\}= \\
= & \sum_{k=0}^{q_{n+3}-m-1}\left|C_{k}^{(n)}\right| .
\end{aligned}
$$

On the other hand

$$
\begin{gathered}
m q_{n+3}^{-1} \leq t \leq(m+1) q_{n+3}^{-1}, \\
1-(m+1) q_{n+3}^{-1} \leq 1-t \leq 1-m q_{n+3}^{-1} .
\end{gathered}
$$

Using the notation $m=q_{n+3}-l-1$, we have

$$
\begin{gathered}
1-\left(q_{n+3}-l-1+1\right) q_{n+3}^{-1} \leq 1-t \leq 1-\left(q_{n+3}-l-1\right) q_{n+3}^{-1} ; \\
l q_{n+3}^{-1} \leq 1-t \leq(l+1) q_{n+3}^{-1}, \quad 1 \leq l<q_{n+1} .
\end{gathered}
$$

From the last inequality we get

$$
\Psi_{n, \theta}(t)=\sum_{k=0}^{l}\left|C_{k}^{(n)}\right|, \quad \text { if } l q_{n+3}^{-1} \leq t \leq(l+1) q_{n+3}^{-1}, \quad 1 \leq l<q_{n+1} .
$$

If $m q_{n+3}^{-1} \leq t \leq(m+1) q_{n+3}^{-1}$ and $q_{n+1} \leq m \leq q_{n+2}$, then

$$
\begin{aligned}
\Psi_{n, \theta}(1-t)=1-\Phi_{n, \theta}(t)= & 1-\left\{\sum_{i=0}^{q_{n+1}-1}\left|B_{i}^{(n)}\right|+\sum_{j=q_{n+2}-m}^{q_{n+2}-1}\left|A_{j}^{(n)}\right|+\sum_{k=q_{n+3}-m}^{q_{n+3}-1}\left|C_{k}^{(n)}\right|\right\}= \\
& =\sum_{j=0}^{q_{n+2}-m}\left|A_{j}^{(n)}\right|+\sum_{k=0}^{q_{n+3}-m}\left|C_{k}^{(n)}\right| .
\end{aligned}
$$

Since, $l q_{n+3}^{-1} \leq 1-t \leq(l+1) q_{n+3}^{-1}$,

$$
\Psi_{n, \theta}(t)=\sum_{j=0}^{l-q_{n+1}}\left|A_{j}^{(n)}\right|+\sum_{k=0}^{l}\left|C_{k}^{(n)}\right|, \quad \text { if } \quad l q_{n+3}^{-1} \leq t \leq(l+1) q_{n+3}^{-1}, \quad q_{n+1} \leq l<q_{n+2} .
$$

If $m q_{n+3}^{-1} \leq t \leq(m+1) q_{n+3}^{-1}, 1 \leq m \leq q_{n+1}$, then

$$
l q_{n+3}^{-1} \leq t \leq(l+1) q_{n+3}^{-1} .
$$

Hence,

$$
\Psi_{n, \theta}(t)=\sum_{i=0}^{l-q_{n+1}}\left|A_{i}^{(n)}\right|+\sum_{j=0}^{l-q_{n+2}}\left|B_{j}^{(n)}\right|+\sum_{k=0}^{l}\left|C_{k}^{(n)}\right|, \text { if } q_{n+2} \leq l<q_{n+3} .
$$

Proposition 5 is completely proved. 


\section{$\S 3$. The proof of Theorem 2}

Fix $k \geq 1$. Consider the sequence of dynamical partitions $\left\{\mathcal{P}_{n-k}\left(x_{0}\right), n \geq k+1\right\}$. By the structure of a dynamical partition, the renormalized neighborhood $V_{n-k}\left(x_{0}\right):=\left[x_{q_{n-k+1}}, x_{q_{n-k}}\right]$ of the critical point $x_{0}$ consists of $q_{k+1}$ intervals of rank $n-1$ and $q_{k}$ intervals of rank $n$. Consider the Poincare first return function $\pi_{n-k}: V_{n-k}\left(x_{0}\right) \rightarrow V_{n-k}\left(x_{0}\right)$ :

$$
\pi_{n-k}(x)= \begin{cases}f^{q_{n-k+1}}(x), & \text { if } x \in\left[x_{0}, x_{q_{n-k}}\right), \\ f^{q_{n-k}}(x), & \text { if } x \in\left[x_{q_{n-k+1}}, x_{0}\right) .\end{cases}
$$

The collection of the following intervals:

$$
\begin{aligned}
& A_{0}^{(n)}, \pi_{n-k}\left(A_{0}^{(n)}\right), \pi_{n-k}^{2}\left(A_{0}^{(n)}\right), \ldots, \pi_{n-k}^{q_{k+2}-1}\left(A_{0}^{(n)}\right) ; \\
& B_{0}^{(n)}, \pi_{n-k}\left(B_{0}^{(n)}\right), \pi_{n-k}^{2}\left(B_{0}^{(n)}\right), \ldots, \pi_{n-k}^{q_{k+1}-1}\left(B_{0}^{(n)}\right) ; \\
& C_{0}^{(n)}, \pi_{n-k}\left(C_{0}^{(n)}\right), \pi_{n-k}^{2}\left(C_{0}^{(n)}\right), \ldots, \pi_{n-k}^{q_{k+3}-1}\left(C_{0}^{(n)}\right),
\end{aligned}
$$

gives the generalized dynamic partition of the renormalized neighborhood $V_{n-k}\left(x_{0}\right)$ associated with the points $x_{0}$ and $c_{n}$. We denote it by $\eta_{n-k, n}\left(x_{0}, c_{n}\right)$. It is clear that the partition $\eta_{n-k, n}\left(x_{0}, c_{n}\right)$ consists of all intervals of the partition $\xi_{n}\left(x_{0}, c_{n}\right)$ contained in the interval $V_{n-k}\left(x_{0}\right)$. To be specific suppose that $n-k$ is even, define the $q_{n}$-small interval $D_{n-k}^{(n)}:=\left[y^{(n)}, z^{(n)}\right) \in \eta_{n-k, n}\left(x_{0}, c_{n}\right)$ and let $D_{n-k}^{(n)} \subset\left[x_{q_{n-k+1}}, x_{0}\right)$. Then $\varphi\left(y^{(n)}\right):=(a, 0, a, 0, \ldots, a, \underline{\varepsilon})$ and $\varphi\left(z^{(n)}\right):=(a, 0, a, 0, \ldots, a, \underline{\gamma})$. The endpoints of the intervals $f^{i}\left(D_{n-k}^{(n)}\right)=\left[f^{i}\left(y^{(n)}\right), f^{i}\left(z^{(n)}\right)\right), 0 \leq i \leq q_{n-k}-1$, have symbolic representation in the form $\varphi\left(f^{i}\left(y^{(n)}\right)\right):=\left(b^{(1)}, b^{(2)}, \ldots, b^{(n-k)}, \underline{\varepsilon}\right)$ and $\varphi\left(f^{i}\left(z^{(n)}\right)\right):=$ $:=\left(b^{(1)}, b^{(2)}, \ldots, b^{(n-k)}, \underline{\gamma}\right)$.

Next define the itinerary of the point $x \in V^{(n-k)}$ as the sequence $\left(b_{1}^{(n-k)}(x), b_{2}^{(n-k)}(x), \ldots\right.$, $\left.\ldots, b_{n}^{n-k)}(x), \ldots\right)$, where $b_{j}^{(n-k)}(x), j \geq 1$, are defined as

$$
b_{j}^{(n-k)}(x):= \begin{cases}0, & \text { if } \pi_{n-k}^{j-1}(x) \in I_{0}^{(n-k+1)}, \\ 1, & \text { if } \pi_{n-k}^{j-1}(x) \in I_{0}^{(n-k)} \backslash\{0\} .\end{cases}
$$

We set

$$
b_{l}^{(n-k)}:=b_{l}\left(C_{0}^{(n)}\right), \quad 1 \leq l \leq q_{k+3} .
$$

We denote by $N_{n-k}^{(i)}(x), i \geq 1$, the $i$-th hitting time of the point $x$ into interval $V_{n-k}$, i.e.,

$$
N_{n-k}^{(i)}(x):=\min \left\{s>i-1: f^{s}(x) \in V_{n-k}\right\} .
$$

It is easy to check that for all $x \in C_{0}^{(n)}$ :

$$
N_{n-k}^{(i)}(x)=\sum_{l=1}^{i}\left(\left(1-b_{l}^{(n-k)}\right) q_{n-k-1}+b_{l}^{(n-k)} q_{n-k}\right), \quad 1 \leq i \leq q_{k+3} .
$$

We denote by $R_{n-k}^{(i)}(x)$ the $i$-th return of $x$ into $V_{n-k}\left(x_{0}\right)$, i. e.,

$$
R_{n-k}^{(i)}(x)=N_{n-k}^{(i)}(x)-N_{n-k}^{(i-1)}(x), \quad i \geq 1 .
$$

It is clear that

$$
R_{n-k}^{(i)}(x)= \begin{cases}q_{n-k}, & \text { if } x \in I_{0}^{(n-k+1)} \\ q_{n-k+1}, & \text { if } x \in I_{0}^{(n-k)}\end{cases}
$$


We define for every $k \geq 1$, the sequence of partitions $\left\{t_{n, i}^{(k)}, 0 \leq i \leq q_{k+3}\right\}_{n=1}^{\infty}$ of the segment $[0,1)$ as follows:

$$
t_{n, i}^{(k)}=\frac{N_{n-k}^{(i)}}{q_{n+3}}, \quad 1 \leq i \leq q_{k+3} \text { and } t_{0}^{(k)}=0 .
$$

Obviously, for each $n \in N$ we have

$$
\begin{gathered}
0 \leq t_{n, 0}^{(1)}<t_{n, 1}^{(1)}<\ldots<t_{n, q_{4}}^{(1)}=1 \\
0 \leq t_{n, 0}^{(2)}<t_{n, 1}^{(2)}<\ldots<t_{n, q_{5}}^{(2)}=1 \\
\ldots \ldots \ldots \ldots \ldots \ldots \ldots \ldots \ldots \ldots t_{n, q_{k+3}}^{(k)}=1
\end{gathered}
$$

Using (3.1) and (3.2), we get the following estimates:

$$
q_{n+3}^{-1} q_{n-k} \leq\left|t_{n, i+1}^{(k)}-t_{n, i}^{(k)}\right| \leq q_{n+3}^{-1} q_{n-k+1}, \quad 0 \leq i \leq q_{k+3}-1 .
$$

One has the following Proposition.

Proposition 6. Let $k>1$ and let the sequence of the partitions $\left\{t_{n, i}^{(k)}, 0 \leq i \leq q_{k+3}\right\}_{n=1}^{\infty}$ of the interval $[0,1)$ be defined by (3.2). Then

1) for each $i, 0 \leq i \leq q_{k+3}-1$, the following finite limit exists:

$$
\lim _{n \rightarrow \infty} t_{n, i}^{(k)}=t_{i}^{(k)}
$$

2) the subset $\mathcal{T}=\bigcup_{k=1}^{\infty}\left\{t_{i}^{k}, 0 \leq i \leq q_{k+3}\right\}$ is dense on $[0,1)$.

P r o o f. It is easy to see that the difference equations $q_{n+1}=q_{n}+q_{n-1}, q_{0}=q_{1}=1$ have solutions

$$
q_{n}=\frac{\bar{\rho}^{2}+\bar{\rho}}{\bar{\rho}^{2}+1} \bar{\rho}^{-n}+(-1)^{n} \frac{1-\bar{\rho}}{\bar{\rho}^{2}+1} \bar{\rho}^{n}, \quad n \geq 0
$$

with $\bar{\rho}=\frac{\sqrt{5}-1}{2}$. Let $1 \leq i \leq q_{k+3}$. Using (3.1)-(3.3) we obtain

$$
\begin{gathered}
t_{n, i}^{(k)}=q_{n+3}^{-1} N_{n-k, n}^{(i)}=q_{n+3} \sum_{l=1}^{i}\left(\left(1-b_{l}^{(k)}\right) q_{n-k-1}+b_{l}^{(k)} q_{n-k}\right)= \\
=\sum_{l=1}^{i}\left(\left(1-b_{l}^{(k)}\right) \bar{\rho}^{k+2}+b_{l}^{(k)} \bar{\rho}^{k+1}+O\left(\bar{\rho}^{2 n-k}\right)\right) .
\end{gathered}
$$

Consequently,

$$
\lim _{n \rightarrow \infty} t_{n, i}^{(k)}=\sum_{l=1}^{i}\left(\left(1-b_{l}^{(k)}\right) \bar{\rho}^{k+2}+b_{l}^{(k)} \bar{\rho}^{k+1}\right)=t_{i}^{(k)}, \quad 1 \leq i \leq q_{k+3} .
$$

Now we prove the second assertion of the Proposition 6. The last relation implies that

$$
c_{1} \bar{\rho}^{k+1} \leq\left|t_{i+1}^{(k)}-t_{i}^{(k)}\right| \leq c_{2} \bar{\rho}^{k}, \quad 0 \leq i \leq q_{k+3},
$$

where the positive constants $c_{1}$ and $c_{2}$ don't depend on $k$ and $i$. Since $\bar{\rho} \in(0,1)$, we obtain that the subset $\mathcal{T}$ is dense on $[0,1]$. 
Proposition 7. For each $i, 0 \leq i \leq q_{k+3}$, there exists the finite limit

$$
\lim _{n \rightarrow \infty} \Psi_{n, \theta}\left(t_{n, i}^{(k)}\right)=\Psi_{\theta}\left(t_{i}^{(k)}\right) .
$$

P r o o f. We rewrite $\Psi_{n, \theta}\left(t_{i}^{(k)}\right)$ in the following form:

$$
\Psi_{n, \theta}\left(t_{i}^{(k)}\right)=\left(\Psi_{n, \theta}\left(t_{i}^{(k)}\right)-\Psi_{n, \theta}\left(t_{n, i}^{(k)}\right)\right)+\Psi_{n, \theta}\left(t_{n, i}^{(k)}\right) .
$$

First, we estimate the difference $\Psi_{n, \theta}\left(t_{i}^{(k)}\right)-\Psi_{n, \theta}\left(t_{n, i}^{(k)}\right)$. The relation (3.2) implies that

$$
\left|t_{i}^{(k)}-t_{n, i}^{(k)}\right| \leq \text { const } \cdot \bar{\rho}^{n-k}, \quad n \geq k+1 .
$$

Using the definition of the distribution function $\Psi_{n, \theta}^{(1)}(t)$ for sufficiently large $n$, we have

$$
\left|\Psi_{n, \theta}\left(t_{i}^{(k)}\right)-\Psi_{n, \theta}\left(t_{n, i}^{(k)}\right)\right| \leq \max _{0 \leq i \leq q_{n}}\left|I_{i}^{(n)}\right| \leq \mathrm{const} \cdot \lambda^{n}
$$

where $\lambda \in(0,1)$. The last term of (3.4) we rewrite in the following form:

$$
\Psi_{n, \theta}\left(t_{n, i}^{(k)}\right)=\sum_{l=1}^{i}\left(\Psi_{n, \theta}\left(t_{n, l}^{(k)}\right)-\Psi_{n, \theta}\left(t_{n, l-1}^{(k)}\right)\right) .
$$

Using definition of the distribution function $\Psi_{n, \theta}$, each difference in the right-hand side of (3.6) can be rewritten as

$$
=\left\{\begin{array}{l}
\Psi_{n, \theta}\left(t_{n, l}^{(k)}\right)-\Psi_{n, \theta}\left(t_{n, l-1}^{(k)}\right)= \\
\sum_{j=0}^{R_{n-k}^{(l)}\left(C_{0}^{(n)}\right)-1}\left|C_{N_{n-k}^{(l-1)}\left(C_{0}^{(n)}\right)+j}^{(n)}\right|, \text { if } 1 \leq l \leq q_{k+1}, \\
\sum_{j=0}^{R_{n-k}^{(l)}\left(A_{0}^{(n)}\right)-1}\left|A_{N_{n-k}^{(l)}\left(A_{0}^{(n)}\right)+j}^{(n)}\right|+\sum_{j=0}^{R_{n-k}^{(l)}\left(C_{0}^{(n)}\right)-1}\left|C_{N_{n-k}^{(l-1)}\left(C_{0}^{(n)}\right)+j}^{(n)}\right|, \text { if } q_{k+1} \leq l<q_{k+2}, \\
\left.\sum_{j=0}^{(l)}\left|B_{N_{n-k}^{(l-1)}\left(B_{0}^{(n)}\right)+j}^{\left(B_{0}^{(n)}\right)-1}\right|+\sum_{j=0}^{R_{n-k}^{(l)}\left(A_{0}^{(n)}\right)-1}\left|A_{N_{n-k}^{(l)}\left(A_{0}^{(n)}\right)+j}^{(l)}\right|+\sum_{j=0}^{(n)} \mid C_{0}^{(n)}\right)-1 \\
\text { if } q_{k+2} \leq l<q_{k+3}^{(n)} .
\end{array}\right.
$$

Each sum in the last relation is the thermodynamic sum of rank $k$ associated with $q_{n}$-small intervals $D^{(n)} \in \eta_{n-k, n}\left(x_{0}, c_{n}\right), D^{(n)} \subset V_{n-k}\left(x_{0}\right)$. Theorem 6 implies that each of such sums has a finite limit as $n \rightarrow \infty$. Futher, collecting (3.4)-(3.6), we obtain, that

$$
\lim _{n \rightarrow \infty} \Psi_{n, \theta}\left(t_{i}^{(k)}\right)=\sum_{l=1}^{i} \lim _{n \rightarrow \infty}\left(\Psi_{n, \theta}\left(t_{n, l}^{(k)}\right)-\Psi_{n, \theta}\left(t_{n, l-1}^{(k)}\right)\right) \equiv \Psi_{\theta}\left(t_{i}^{(k)}\right)
$$

for all $1 \leq i \leq q_{k+3}$.

It remains to prove the continuity of $\Psi_{\theta}(t)$ on $[0,1]$. Since $\Psi_{\theta}(t)$ is increasing function on the dense subset $\mathcal{T}$, it can be extended to the interval $[0,1)$. It is obvious, that $\Psi_{\theta}(t)=0$, if $t \leq 0$, and $\Psi_{\theta}(t)=1$, if $t \geq 1$. We clearly deduce that

$$
\lim _{n \rightarrow \infty} \Psi_{n, \theta}(t)=\Psi_{\theta}(t)
$$


for all $t \in \mathbb{R}^{1}$.

Now we prove continuity of the limit distribution function $\Psi_{\theta}(t)$ on $[0,1]$. By the triangle inequality, we have

$$
\begin{aligned}
\left|\Psi_{\theta}\left(t_{i+1}^{(k)}\right)-\Psi_{\theta}\left(t_{i}^{(k)}\right)\right| \leq & \left|\Psi_{\theta}\left(t_{i+1}^{(k)}\right)-\Psi_{n, \theta}\left(t_{i+1}^{(k)}\right)\right|+\left|\Psi_{\theta}\left(t_{i}^{(k)}\right)-\Psi_{n, \theta}\left(t_{i}^{(k)}\right)\right|+ \\
& +\left|\Psi_{n, \theta}\left(t_{i+1}^{(k)}\right)-\Psi_{n, \theta}\left(t_{n, i}^{(k)}\right)\right| .
\end{aligned}
$$

Now we estimate the last sum in (3.7). We consider only the case $0 \leq i \leq q_{k+1}$. Other cases are similar. We have:

$$
\left|\Psi_{n, \theta}\left(t_{n, i}^{(k)}\right)-\Psi_{n, \theta}\left(t_{n, i-1}^{(k)}\right)\right| \leq \sum_{j=0}^{R_{n-k}^{(i)}\left(C_{0}^{(n)}\right)-1}\left|I_{j}^{(n-k)}\right| \cdot \frac{\left|C_{N_{n-k}^{(n)}\left(C_{0}^{(n)}\right)+j}^{(n)}\right|}{\left|I_{j}^{(n-k)}\right|} \leq \mathrm{const} \cdot \lambda^{n}
$$

here we used that the $q_{n}$-small interval $C_{N_{n-k}^{(l-1)}\left(C_{0}^{(n)}\right)+j}^{(n)}$ is the part of the interval $I_{j}^{(n-k)}$ of rank $n-k$.

Take an arbitrary small $\varepsilon>0$. We choose $k=k(\varepsilon)>0$ such that const $\cdot \lambda^{n}<\frac{\varepsilon}{6}$. However the sequence $\left\{\Psi_{n, \theta}(t)\right\}$ converges to $\Psi_{\theta}(t)$, for sufficiently large $n$ the sum of first two terms in the right-hand side of the inequality (3.7) is less than $\frac{\varepsilon}{6}$ for all $0 \leq i \leq q_{k+3}$. From (3.5), we have that for sufficiently large $n$ the sum of next two terms is less than $\frac{\varepsilon}{6}$. We collect all above estimates and obtain that the right-hand side of (3.8) is less than $\frac{\varepsilon}{2}$ for all $0 \leq i \leq q_{k+3}$. Put $\delta=\alpha^{k-1}$. Since the function $\Psi_{\theta}(t)$ is increasing, then using (3.7) and (3.8), we obtain that $\left|\Psi_{\theta}\left(t_{1}\right)-\Psi_{\theta}\left(t_{2}\right)\right|<\varepsilon$, if $\left|t_{2}-t_{1}\right|<\delta$.

The last statement of Theorem 2 can be proved as a similar statement in [21].

\section{REFERENCES}

1. de Faria E., de Melo W. Rigidity of critical circle mappings. I, Journal of the European Mathematical Society, 1999, vol. 1, issue 4, pp. 339-392. https://doi.org/10.1007/s100970050011

2. Ostlund R., Rand D., Sethna J., Siggia E. Universal properties of the transition from quasi-periodicity to chaos in dissipative systems, Physica D: Nonlinear Phenomena, 1983, vol. 8, issue 3, pp. 303-342. https://doi.org/10.1016/0167-2789(83)90229-4

3. Lanford O. E., III, de la Llave R. Solution of the functional equation for critical circle mappings with golden rotation number. Manuscript, 1984.

4. Epstein H. Fixed points of composition operators. II, Nonlinearity, 1989, vol. 2, no. 2, pp. 305-310. https://doi.org/10.1088/0951-7715/2/2/006

5. de Melo W., van Strien S. One-dimensional dynamics, Berlin: Springer, 1993. https://doi.org/10.1007/978-3-642-78043-1

6. Mestel B. A computer assisted proof of universality for Cubic critical maps of the circle with Golden mean rotation number, $\mathrm{PhD}$ thesis, University of Warwick, 1984.

7. Lanford O.E., III. Functional equations for circle homeomorphisms with golden ratio rotation number, Journal of Statistical Physics, 1984, vol. 34, no. 1-2, pp. 57-73. https://doi.org/10.1007/BF01770349

8. Yoccoz J.-Ch. Il n'y a pas de contre-exemple de Denjoy analytique, Comptes Rendus de l'Académie des Sciences. Série I Mathématique, 1984, vol. 298, no. 7, pp. 141-144. https://zbmath.org/?q=an:0573.58023

9. Graczyk J., Światek G. Singular measures in circle dynamics, Communications in Mathematical Physics, 1993, vol. 157, no. 2, pp. 213-230. https://doi.org/10.1007/BF02099758

10. Guarino P., Martens M., de Melo W. Rigidity of critical circle maps, Duke Mathematical Journal, 2018, vol. 167, no. 11, pp. 2125-2188. https://doi.org/10.1215/00127094-2018-0017

11. Coelho Z., de Faria E. Limit laws of entrance times for homeomorphisms of the circle, Israel Journal of Mathematics, 1996, vol. 93, no. 1, pp. 93-112. https://doi.org/10.1007/BF02761095 
12. Coelho Z. The loss of tightness of time distributions for homeomorphisms of the circle, Transactions of the American Mathematical Society, 2004, vol. 356, no. 11, pp. 4427-4445. https://doi.org/10.1090/S0002-9947-04-03386-0

13. Kim D. H., Seo B. K. The waiting time for irrational rotations, Nonlinearity, 2003, vol. 16, no. 5, pp. 1861-1868. https://doi.org/10.1088/0951-7715/16/5/318

14. Katznelson Y., Ornstein D. The absolute continuity of the conjugation of certain diffeomorphisms of the circle, Ergodic Theory and Dynamical Systems, 1989, vol. 9, no. 4, pp. 681-690. https://doi.org/10.1017/S0143385700005289

15. Pitskel B. Poisson limit law for Markov chains, Ergodic Theory and Dynamical Systems, 1991, vol. 11, no. 3, pp. 501-513. https://doi.org/10.1017/S0143385700006301

16. Bowen R. E. Equilibrium states and the ergodic theory of Anosov diffeomorphisms. Berlin: Springer, 2008. https://doi.org/10.1007/978-3-540-77695-6

17. Collet P., Galves A. Asymptotic distribution of entrance times for expanding maps of the interval, Dynamical Systems and Applications, World Scientific, 1995, pp. 139-152. https://doi.org/10.1142/9789812796417_0011

18. Vul E. B., Sinai Ya.G., Khanin K.M. Feigenbaum universality and the thermodynamic formalism, Russian Mathematical Surveys, 1984, vol. 39, no. 3, pp. 1-40. https://doi.org/10.1070/RM1984v039n03ABEH003162

19. Dzhalilov A. A. Thermodynamic formalism and singular invariant measures for critical circle maps, Theoretical and Mathematical Physics, 2003, vol. 134, no. 2, pp. 166-180. https://doi.org/10.1023/A:1022271903129

20. Ruelle D. Thermodynamic formalism. The mathematical structure of equilibrium statistical mechanics, Cambridge: Cambridge University Press, 2004. https://doi.org/10.1017/CBO9780511617546

21. Dzhalilov A.A. Limiting laws for entrance times of critical mappings of a circle, Theoretical and Mathematical Physics, 2004, vol. 138, no. 2, pp. 190-207.

https://doi.org/10.1023/B:TAMP.0000014851.67668.fb

Received 24.02.2021

Shavkat Abdullaevich Ayupov, Doctor of Physics and Mathematics, Academician, Institute of Mathematics of ASRUz, Tashkent, Uzbekistan.

ORCID: https://orcid.org/0000-0002-4006-1460

E-mail: sh_ayupov@mail.ru

Alisher Akbarovich Zhalilov, PhD Candidate, Department of Exact Science, Yeoju Technical Institute in Tashkent, Tashkent, Uzbekistan; Institute of Mathematics of ASRUz, Tashkent, Uzbekistan.

ORCID: https://orcid.org/0000-0002-7105-1812

E-mail: adjalilov2013@gmail.com

Citation: Sh. A. Ayupov, A. A. Zhalilov. Asymptotic distribution of hitting times for critical maps of the circle, Vestnik Udmurtskogo Universiteta. Matematika. Mekhanika. Komp'yuternye Nauki, 2021, vol. 31, issue 3, pp. 365-383. 


\section{ШІ. А. Аюпов, А. А. Жалилов \\ Асимптотическое распределение времени попадания для критических отображений на окруж- ности}

Ключевые слова: гомеоморфизм круга, критическая точка, число вращения, время попадания, термодинамический формализм.

УДК 517.9

\section{DOI: $10.35634 / \mathrm{vm} 210302$}

Хорошо известно, что преобразование ренормгруппы $\mathcal{R}$ имеет единственную неподвижную точку $f_{c r}$ в пространстве критических $C^{3}$-гомеоморфизмов окружности с одной кубической критической точкой $x_{c r}$ и числом вращения, равным золотому сечению $\bar{\rho}:=\frac{\sqrt{5}-1}{2}$. Обозначим через $C r(\bar{\rho})$ множество всех критических отображений окружности, $C^{1}$-сопряженных к $f_{c r}$. Пусть $f \in C r(\bar{\rho})$ и $\mu:=\mu_{f}-$ единственная вероятностная инвариантная мера для $f$. Зафиксируем $\theta \in(0,1)$. Для каждого $n \geqslant 1$ определим $c_{n}:=c_{n}(\theta)$ такое, что $\mu\left(\left[x_{c r}, c_{n}\right]\right)=\theta \cdot \mu\left(\left[x_{c r}, f^{q_{n}}\left(x_{c r}\right)\right]\right)$, где $q_{n}-$ время первого возврата линейного вращения $f_{\bar{\rho}}$. Мы исследуем закон сходимости перемасштабированного точечного процесса времени попадания. Мы показываем, что предельное распределение сингулярно относительно меры Лебега.

\section{СПИСОК ЛИТЕРАТУРЫ}

1. de Faria E., de Melo W. Rigidity of critical circle mappings. I // Journal of the European Mathematical Society. 1999. Vol. 1. Issue 4. P. 339-392. https://doi.org/10.1007/s100970050011

2. Ostlund R., Rand D., Sethna J., Siggia E. Universal properties of the transition from quasi-periodicity to chaos in dissipative systems // Physica D: Nonlinear Phenomena. 1983. Vol. 8. Issue 3. P. 303-342. https://doi.org/10.1016/0167-2789(83)90229-4

3. Lanford O.E., III, de la Llave R. Solution of the functional equation for critical circle mappings with golden rotation number. Manuscript, 1984.

4. Epstein H. Fixed points of composition operators. II // Nonlinearity. 1989. Vol. 2. No. 2. P. 305-310. https://doi.org/10.1088/0951-7715/2/2/006

5. de Melo W., van Strien S. One-dimensional dynamics. Berlin: Springer, 1993. https://doi.org/10.1007/978-3-642-78043-1

6. Mestel B. A computer assisted proof of universality for Cubic critical maps of the circle with Golden mean rotation number. PhD thesis, University of Warwick, 1984.

7. Lanford O.E., III. Functional equations for circle homeomorphisms with golden ratio rotation number // Journal of Statistical Physics. 1984. Vol. 34. No. 1-2. P. 57-73. https://doi.org/10.1007/BF01770349

8. Yoccoz J.-Ch. Il n'y a pas de contre-exemple de Denjoy analytique // Comptes Rendus de l'Académie des Sciences. Série I Mathématique. 1984. Vol. 298. No. 7. P. 141-144. https://zbmath.org/?q=an:0573.58023

9. Graczyk J., Światek G. Singular measures in circle dynamics // Communications in Mathematical Physics. 1993. Vol. 157. No. 2. P. 213-230. https://doi.org/10.1007/BF02099758

10. Guarino P., Martens M., de Melo W. Rigidity of critical circle maps // Duke Mathematical Journal. 2018. Vol. 167. No. 11. P. 2125-2188. https://doi.org/10.1215/00127094-2018-0017

11. Coelho Z., de Faria E. Limit laws of entrance times for homeomorphisms of the circle // Israel Journal of Mathematics. 1996. Vol. 93. No. 1. P. 93-112. https://doi.org/10.1007/BF02761095

12. Coelho Z. The loss of tightness of time distributions for homeomorphisms of the circle // Transactions of the American Mathematical Society. 2004. Vol. 356. No. 11. P. 4427-4445. https://doi.org/10.1090/S0002-9947-04-03386-0

13. Kim D. H., Seo B. K. The waiting time for irrational rotations // Nonlinearity. 2003. Vol. 16. No. 5. P. 1861-1868. https://doi.org/10.1088/0951-7715/16/5/318 
14. Katznelson Y., Ornstein D. The absolute continuity of the conjugation of certain diffeomorphisms of the circle // Ergodic Theory and Dynamical Systems. 1989. Vol. 9. No. 4. P. 681-690. https://doi.org/10.1017/S0143385700005289

15. Pitskel B. Poisson limit law for Markov chains // Ergodic Theory and Dynamical Systems. 1991. Vol. 11. No. 3. P. 501-513. https://doi.org/10.1017/S0143385700006301

16. Bowen R. E. Equilibrium states and the ergodic theory of Anosov diffeomorphisms. Berlin: Springer, 2008. https://doi.org/10.1007/978-3-540-77695-6

17. Collet P., Galves A. Asymptotic distribution of entrance times for expanding maps of the interval // Dynamical Systems and Applications. World Scientific, 1995. P. 139-152. https://doi.org/10.1142/9789812796417_0011

18. Vul E. B., Sinai Ya. G., Khanin K. M. Feigenbaum universality and the thermodynamic formalism // Russian Mathematical Surveys. 1984. Vol. 39. No. 3. P. 1-40. https://doi.org/10.1070/RM1984v039n03ABEH003162

19. Dzhalilov A. A. Thermodynamic formalism and singular invariant measures for critical circle maps // Theoretical and Mathematical Physics. 2003. Vol. 134. No. 2. P. 166-180. https://doi.org/10.1023/A:1022271903129

20. Ruelle D. Thermodynamic formalism. The mathematical structure of equilibrium statistical mechanics. Cambridge: Cambridge University Press, 2004. https://doi.org/10.1017/CBO9780511617546

21. Dzhalilov A. A. Limiting laws for entrance times of critical mappings of a circle // Theoretical and Mathematical Physics. 2004. Vol. 138. No. 2. P. 190-207. https://doi.org/10.1023/B:TAMP.0000014851.67668.fb

Поступила в редакцию 24.02 .2021

Аюпов Шавкат Абдуллаевич, д.ф.-м. н., академик АН Узбекистана, Институт математики имени В. И. Романовского Академии наук Республики Узбекистан, 100077, Узбекистан, г. Ташкент, ул. Мирзо Улугбека, 81.

ORCID: https://orcid.org/0000-0002-4006-1460

E-mail: sh_ayupov@mail.ru

Жалилов Алишер Акбарович, аспирант, Йоджинский технический институт в Ташкенте, Узбекистан, Ташкент;

Институт математики имени В. И. Романовского Академии наук Республики Узбекистан, 100077, Узбекистан, г. Ташкент, ул. Мирзо Улугбека, 81.

ORCID: https://orcid.org/0000-0002-7105-1812

E-mail: adjalilov2013@gmail.com

Цитирование: Ш.А. Аюпов, А. А. Жалилов. Асимптотическое распределение времени попадания для критических отображений на окружности // Вестник Удмуртского университета. Математика. Механика. Компьютерные науки. 2021. Т. 31. Вып. 3. С. 365-383. 